\title{
Deraining and Desnowing Algorithm on Adaptive Tolerance and Dual-tree Complex Wavelet Fusion
}

\author{
Jingfeng Zang*, Ningxue Xu, Rui Liu, Yuhuan Shi \\ Changchun University of Science and Technology, College of Electronic and Information Engineering, \\ No. 7089 Weixing Road, Changchun, 130022 (China)
}

Received 16 May 2020 | Accepted 28 October 2020 | Published 25 November 2020

\section{ABSTRACT}

Severe weather conditions such as rain and snow often reduce the visual perception quality of the video image system, the traditional methods of deraining and desnowing usually rarely consider adaptive parameters. In order to enhance the effect of video deraining and desnowing, this paper proposes a video deraining and desnowing algorithm based on adaptive tolerance and dual-tree complex wavelet. This algorithm can be widely used in security surveillance, military defense, biological monitoring, remote sensing and other fields. First, this paper introduces the main work of the adaptive tolerance method for the video of dynamic scenes. Second, the algorithm of dual-tree complex wavelet fusion is analyzed and introduced. Using principal component analysis fusion rules to process low-frequency sub-bands, the fusion rule of local energy matching is used to process the high-frequency sub-bands. Finally, this paper used various rain and snow videos to verify the validity and superiority of image reconstruction. Experimental results show that the algorithm has achieved good results in improving the image clarity and restoring the image details obscured by raindrops and snows.

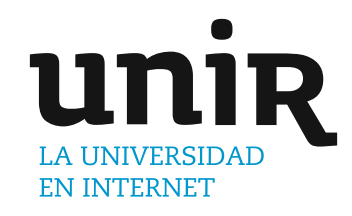

KEYWORDS

Adaptive Tolerance,

Dual-tree Complex

Wavelet Fusion,

Deraining And

Desnowing, Video

Enhancement.

DOI: $10.9781 /$ ijimai.2020.11.014

\section{INTRODUCTION}

$\mathrm{T}$ HE video image captured by the outdoor video image system in severe weather (rain and snow) is severely degraded. Generally, the color and contrast of captured images and videos will drop sharply, which greatly affects the normal operation of outdoor video image systems, such as security surveillance systems, video recognition, intelligent perception, environmental safety, and intelligent transportation. Because there is a sequence of feature information in the video image, the research on the video image rain and snow removal algorithm is the main research direction in this field. Videobased image rain and snow removal algorithms are roughly divided into several types of algorithms based on the time domain, space domain, and color characteristics. Among them, the algorithm based on the image space domain started earlier and the research is more in-depth.

For example, Hase [1] and others use the size and speed of moving objects to propose a new method that can remove snow noise from the continuous images recorded by the camera. Garg [2]-[3] and others conducted a comprehensive analysis of the visual impact of the rain imaging system for the first time, and proposed to analyze the motion and optical characteristics of rain and snow, calculated the dynamics and optical models of rain and snow to identify and process the frame difference method of rain and snow. Camera parameters were used to reduce the visibility of rain during image acquisition, but the removal effect of this algorithm is not ideal in the case of heavy rain and snow

* Corresponding author.

E-mail address: zjf0319830@sohu.com scenes and when there are high-speed moving objects in degraded images. Zhang et al. [4] proposed a k-means clustering method for all the same coordinates pixels along the video timeline, which can detect and clear away raindrops, this method is proposed based on the combination of the time and chroma characteristics of raindrops in the video. The clustering method needs to be applied to the entire video sequence, and this method cannot be used online. Then through the improved frame difference method or clustering method they achieve image space to remove rain (snow). Brewer et al. [5] adopted a multi-step method to eliminate rainwater and similar effects in video clips, a new method of raindrop pixel replacement is constructed by detecting raindrops in five consecutive frames. Zhao Xudong et al. [6] proposed an improved histogram model for detecting and removing rainwater in video, using the brightness distribution range of raindrops to determine whether pixels are covered by raindrops. Barnum et al. [7]-[8] combined the fringe model and rain and snow statistical characteristics to create an overall effect model of dynamic weather in frequency space, suitable for video detection and rain and snow removal with scenes and camera motion, but the model construction process is more complicated. Bossu et al. [9] proposed to use the classic Gaussian mixture model to segregate the foreground model from the image sequence for detecting rain and snow. Based on the choice rules of luminosity and size, the geometric moment method is used to estimate the histogram of the rain and snow stripe direction, and the expectation-maximization algorithm is used to achieve the purpose of removing rainwater in the video image. Kang et al. [10][11] proposed for the first time that a single image can be denoised by a sparse coding algorithm and morphological component analysis model. Then,they used the time information of consecutive frames and reused the dictionary learned in the previous frame of the video, while 
holding video time consistency, frame-by-frame image deraining, the rain removal based on the video is an extension of the rain removal method of a single image, but this method is only applicable to static scenes. Yu [12] proposed a video defogging technique based on adaptive tolerance, and combined it with the guiding filter to apply video defogging. Abdel-Hakim [13] used inter-frame information to recover low-rank components between adjacent frames to extract rain-free distortion signals, and used low-rank recovery methods to remove raindrops and snowflakes from the video frames. Kim et al. [14] proposed a new algorithm for removing rain and snow stripes through low-rank matrix video sequences, and extended this algorithm to three-dimensional stereo video cancellation, but when processing dynamic video images, the snow removal effect is not ideal. Ren et al. [15] proposed a video denoising and drainage model based on matrix decomposition, which solved the problem that the rain and snow removal method could not cope with heavy rain, blizzard and dynamic scenes. Zheng et al. [16] proposed a method of through multiguide filter in a single image to remove raindrops and snowflakes, the low-frequency sub-band obtained by decomposition is a non-raindrop and snowflake image, and the high-frequency sub-band is input to the guide filter as an input image. Wang et al. [17] proposed an algorithm that uses wavelet fusion technology to achieve video rain removal, combined with wavelet multi-layer decomposition technology to determine the particular layer of rain noise. L. Dou et al. [20] improved the traditional dual-tree complex wavelet image restoration method and proposed a new algorithm. The image is decomposed into two parts of low-frequency and high-frequency using the complex wavelet transform, the high-frequency components of the image are repaired by the total change model, and the improved curvature-driven diffusion is used to repair the low-frequency components. J. Zang et al. [31] improved on the traditional dual-tree complex wavelet and proposed an improved method to eliminate raindrops, this method uses the fusion rule of principal component analysis for the low-frequency sub-bands obtained by the complex wavelet decomposition, and uses local energy matching for the high-frequency sub-bands fusion rules.

On this basis, aiming at the problem of distortion in bright areas of fusion images under more complicated rain and snow weather backgrounds, this paper proposes a method to eliminate video rain and snow by combining adaptive tolerance and dual-tree complex wavelet. The adjustment mechanism based on adaptive tolerance can not only solve the problem of distortion in bright areas, but also not cause transmittance. The error compensation is obtained, so that a higher quality fusion image can be restored. At the same time, the video image is decomposed by dual-tree complex wavelet, and different sub-images are formulated with different fusion rules to improve the quality of the video image under the complex weather background. The performance of this method has been improved and is better than the methods already proposed.

\section{Video Enhancement Based on Adaptive Tolerance}

\section{A. Video Enhancement of Dynamic Scene}

For dynamic scene videos which are caused by camera motion, each frame of the video is enhanced as a single image in our method, which requires the processing rate of each video frame to be fast enough. Firstly, to estimate the rough transmission amount of each frame by the dark prior channel, the guide filter is used to refine the rough transmission amount quickly. Then it is needed to be judged whether a large bright region exists in the intercepted video frame. If [12] it exists, the transmission requires to be corrected with the help of the adaptive tolerance, if not, the transmission can be directly used to recover the scene radiance. The flowchart of the video enhancement based on adaptive tolerance is shown in Fig. 1.

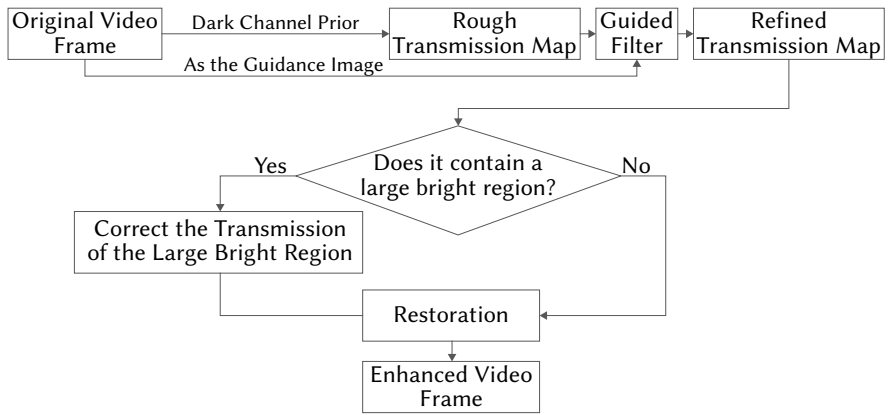

Fig. 1. Flowchart of video enhancement based on adaptive tolerance.

\section{B. Dark Channel Prior and Rough Transmission Map}

The [18] dark channel prior can be understood as a statistic for outdoor fog-free images. Most of the local areas in outdoor fog-free images will contain some very dark pixels, which exist in at least one color channel. The min operation is taken to both the local patch and three color channels, and it is obtained:

$$
\min _{k \in \Omega(l)}\left(I^{C}(k)\right)=\tilde{t}(l) \min _{k \in \Omega(l)}\left(J^{C}(k)\right)+(1-\tilde{t}(l)) A^{C}
$$

$l$ and $k$ respectively refer to image pixels and local area pixels, $t(l)$ is the transmittance, and A is the atmospheric light value, $\Omega(I)$ is a local area window centered on 1 . The purpose of this method is to restore the fog-free image J from the fog-free image I. Then the dark channel of the source image is obtained by:

$$
J^{\text {dark }}(l)=\min _{C}\left(\min _{k \in \Omega(l)}\left(J^{C}(k)\right)\right) \rightarrow 0
$$

the rough transmittance can be estimated as follows:

$$
\tilde{t}(l)=1-\omega \min _{C}\left(\min _{k \in \Omega(l)}\left(\frac{I^{C}(k)}{A^{C}}\right)\right)
$$

Select the pixel with the highest fog density in the dark channel (the first $0.1 \%$ of the brightest), and then select the pixel with the highest intensity among the pixels corresponding to this point in the source fog image as A. Fig. 3 shows the rough transmission map estimated from Fig. 2.

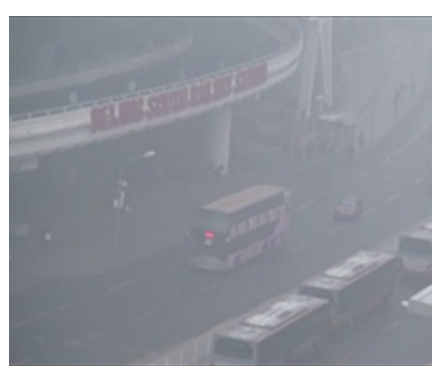

Fig. 2. Original video frame.

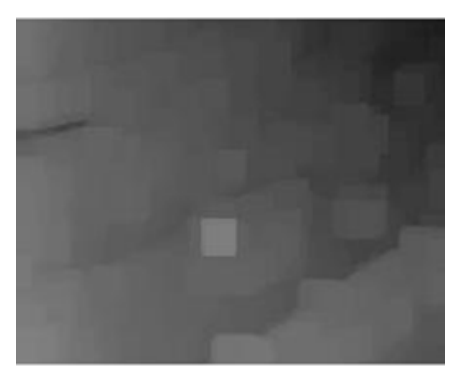

Fig. 3. Rough transmission map.

\section{The Guided Filter and Refined Transmission Map}

If the rough transmission is directly used to recover the enhanced image, halo artifacts will appear at the edges with discontinuous depth. Therefore, a guided filter is introduced to refine the rough transmittance. The refined transmittance is defined as:

$$
t(y)=a_{l}^{T} I(k)+b_{l} \quad \forall k \in \omega_{l}
$$

In the local window $\omega_{l}$, there is a linear relationship between $I(k)$ and $t(k)$, and the coefficient $a_{l}$ and $b_{l}$ is defined as:

$$
\begin{aligned}
& a_{l}=\left(\sum_{l}+\varepsilon U\right)^{-1}\left(\frac{1}{|\omega|} \sum_{k \in \omega_{l}} I(k) \tilde{t}(k)-\mu_{l} \bar{t}(l)\right) \\
& b_{l}=\bar{t}(l)-a_{l}^{T} \mu_{l}
\end{aligned}
$$


Refer to (5)-(6), Equation (4) indicates that refined transmission can obtain the refined transmittance for each frame. Refining the transmission by the guided filter is not only efficient, but can also produce good results comparable with the results of soft matting. The result is shown in Fig. 4.

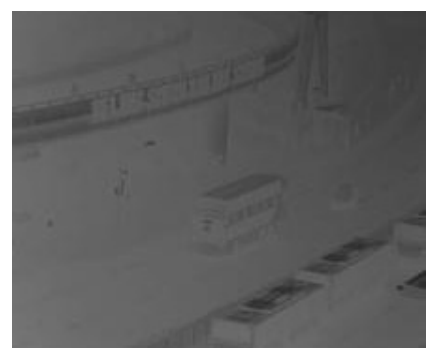

Fig. 4. Refined transmission map.

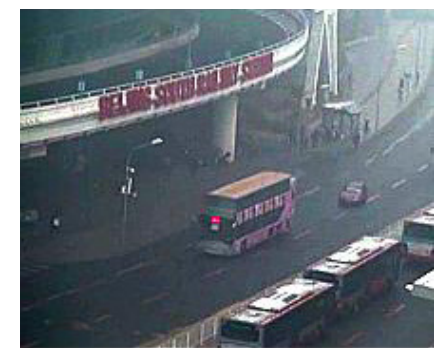

Fig. 5. Restored video frame, $\mathrm{U}_{\max }=50$.

\section{The Image Restoration Based on the Adaptive Tolerance}

The [12] inaccurate transmission estimated can be corrected according to the similarity degree between the source image $I$ and the atmospheric light $A$. If a region of the source image satisfies that the value of $|I-A|$ is smaller than or equal to the tolerance, the transmission of the region considered to be a bright region will be recalculated. The region whose value of $|I-A|$ is bigger than the tolerance keeps its transmission unchanged, because it is considered to the region which meets the dark channel prior. Tolerance value can be determined adaptively according to the histogram of the transmission of the original video frame, which meets the requirement of automaticity in video enhancement, and the color distortion can be avoided effectively in image restoration.

Thus, if the original video frame contains a large bright region, it is needed to search out the first maximum point $\left(U_{\max } P_{\max }\right)$ on the left side of the histogram curve of the refined transmission map. Equation (7) can be used to correct transmission by substituting $U_{\max }$. According to the value of $|I-A|$ in each color channel, the three corrected transmission maps $q^{C}(I)$ can be separately used to recover the three color channels of the enhanced image. When $U_{\max }$ was set to be ' 50 ', the restored video frame is shown in Fig. 5.

$$
\begin{aligned}
& q^{C}(l)=\min \left(\max \left(\frac{U_{\max }}{\left|I^{C}(l)-A^{C}\right|}, 1\right) \cdot \max \left(t(l), t_{0}\right), 1\right) \\
& c \in\{r, g, b\}
\end{aligned}
$$

$U_{\max }$ should be set to be ' 0 ', if the original video frame does not contain a large bright region, and the transmission will not be corrected.

\section{Image Fusion Based on Dual-Tree Complex Wavelet}

\section{A. Dual-tree Complex Wavelet}

The dual-tree complex wavelet transform adopts a binary tree structure, and the signal is decomposed and reconstructed by two independent real number filter banks. The complex wavelet transform is realized by a real wavelet transform. The problem that the complex wavelet transform cannot be completely reconstructed is solved.

Among [21] them, the one-dimensional complex wavelet function is represented by Equation (8):

$$
\psi(\alpha)=\psi_{h}(\alpha)+i \psi_{g}(\alpha)
$$

the two-dimensional complex wavelet function is expressed by Equation (9):

$$
\psi(\alpha, \beta)=\psi(\alpha) \psi(\beta)=\left[\psi_{h}(\alpha)+i \psi_{g}(\alpha)\right]\left[\psi_{h}(\beta)+i \psi_{g}(\beta)\right]
$$

Use $R[\psi(\alpha, \beta)]$ to represent the real part and $I[\psi(\alpha, \beta)]$ to represent the imaginary part:

$$
\begin{aligned}
& R[\psi(\alpha, \beta)]=\psi_{h}(\alpha) \psi_{h}(\beta)-\psi_{g}(\alpha) \psi_{g}(\beta) \\
& I[\psi(\alpha, \beta)]=\psi_{g}(\alpha) \psi_{h}(\beta)+\psi_{h}(\alpha) \psi_{g}(\beta)
\end{aligned}
$$

The discrete wavelet transform can only get three sub-bands in different directions for each layer of decomposition. However [20], each layer of dual-tree complex wavelet transform can be decomposed to obtain 6 sub-bands, as shown in Fig. 6. This method improves the accuracy of image decomposition and reconstruction, and can retain more detailed information about the image.

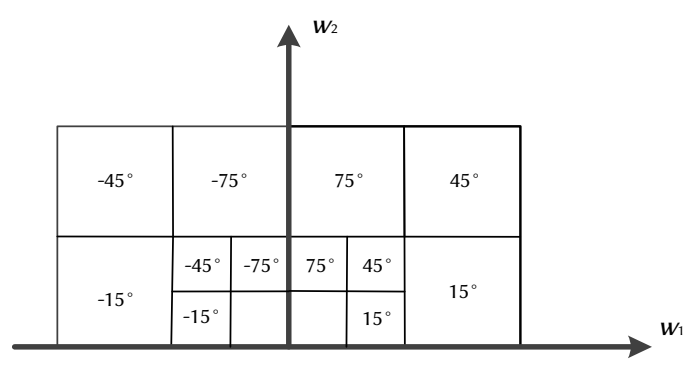

Fig. 6. Direction Sub-images Distribution.

\section{B. Image Fusion}

The procedure of fusing together several images in the same scene collected by different sensors to obtain an image with complementary information is called image fusion. Fig. 7 shows the main process of image fusion. First, the input image is preprocessed, mainly the process of noise reduction and correction, then the processed image is matched with features, and finally, image fusion is performed. The most basic and common image preprocessing technique is image enhancement. Image [22] enhancement is mainly for specific processing of images, obtaining new images with better visual quality. Image matching is a process of aligning multiple images obtained at different times, different viewing angles or different sensor devices. Through the matching algorithm, the similarity and consistency of the image are found in textures, features and structures, then similar images are obtained. Image [23] fusion is widely used in security surveillance, military defense, biological monitoring, remote sensing and other fields.

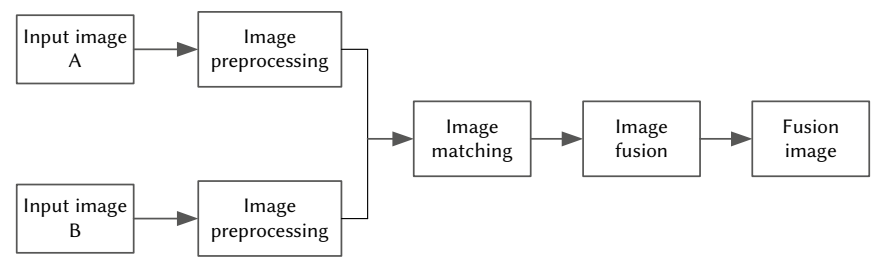

Fig. 7. Image Fusion flow chart.

\section{The Proposed Video Deraining and Desnowing Algorithm}

The deraining and desnowing algorithm on dual-tree complex wavelet fusion and adaptive tolerance proposed in this paper is mainly divided into three modules to complete: improved image preprocessing based on adaptive tolerance, optimized image feature matching based on SIFT and dual-tree complex Image fusion of wavelet. Fig. 8 shows the overall flow chart of the fusion algorithm. 


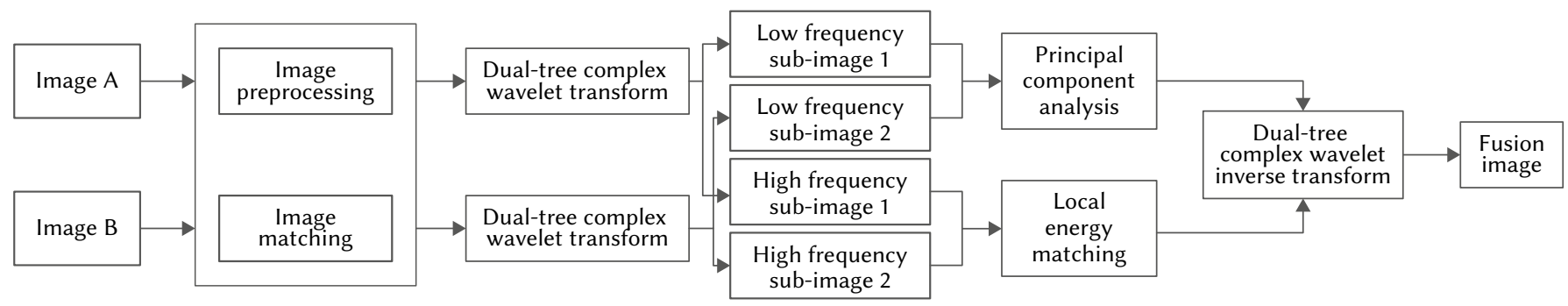

Fig. 8. The proposed algorithm flow chart.

\section{A. Image Preprocessing Based on Adaptive Tolerance}

The image acquired by the sensor usually weakens the contrast, which makes the edge and contour information in the image appear inconspicuous to a certain extent, and the imaging effect becomes blurred. In order to solve the problem of bright area distortion in traditional image preprocessing algorithms, this article [24] proposes an improved image preprocessing algorithm based on adaptive tolerance in the chapter 'Video enhancement based on adaptive tolerance'. It can adaptively determine the tolerance parameters of the bright area according to the image, correct the transmittance value of the bright area, and effectively reduce the error compensation of the transmittance, maximize the reduction of image distortion after deraining and desnowing, and improve the quality of video images quality after deraining and desnowing.

\section{B. Image Matching}

The SIFT algorithm is the mainstream algorithm for image feature matching, which has far and wide application on the seeking of key points, remaining the immutability and stability of brightness, translation, rotation, and scale, in different scale-spaces. To calculate the Euclidean distance between the two sets of feature points, the method of using feature points to extract feature descriptors is applied first, and then the match between feature descriptors is apparently figured out. The smaller the Euclidean distance, the higher the similarity. When the Euclidean distance is less than a certain threshold, the match is considered as a success. The SIFT algorithm of MLESAC is outstandingly proposed in [25] and turned into a basis of the improvement in [20]. The approach involves three steps:

The first step is to detect and extract the feature points of the preprocessed image and the reference image.

The second is a rough match. The Euclidean distance of the key point feature vector is used for rough matching to generate unmatched feature points.

The last step is exact matching. For the feature point pairs matched roughly in the second procedure, the algorithm [25] applies to statistical estimation. After excluding the mismatching feature points, exact matching of the feature points happens with eventual success.

\section{Image Fusion Based on Dual-tree Complex Wavelet}

The [26] fusion image obtained based on the discrete wavelet transform fusion algorithm usually has artifact effects on the edges. To [27] solve the above problems, this paper improves the traditional dual-tree complex wavelet, different from wavelet transform, dualtree complex wavelet transform has good direction selectivity and reduces the translation sensitivity of wavelet transform. The [20] fusion process is divided into the following three modules:

\section{(1) Decompose the input image}

The dual-tree complex wavelet transform first decomposes in the row direction and then decomposes in the column direction to obtain two low-frequency sub-bands denoted as $I_{A}{ }^{N}(p, q)$ and $I_{B}{ }^{N}(p, q)$, and six high-frequency sub-bands $\left\{I_{A}^{l . k}(p, q) 、 I_{B}^{l, k}(p, q)\right\}$ in different directions.

\section{(2) Sub-image fusion}

The low-frequency images after wavelet decomposition display the overall characteristics of the image and contains some significant details (such as edges and contours). The selection of which fusion rule directly effects the consequence of image fusion. In order to obtain a clearer image fusion result, this paper adopts the weighting criterion and uses principal component analysis to fuse low-frequency images. The [27] fusion process is as follows:

$$
I_{L F}{ }^{N}(p, q)=w_{A} \times I_{A}{ }^{N}(p, q)+w_{B} \times I_{B}{ }^{N}(p, q)
$$

$I_{A}{ }^{N}(p, q)[29]$ and $I_{B}{ }^{N}(p, q)$ respectively represent the low-frequency coefficients of the source image at the baseband (p, q) position, $(x, y)^{T}$ are their corresponding feature vectors, then the weighting coefficient is defined as:

$$
w_{A}=\frac{x}{x+y}, w_{B}=\frac{y}{x+y}
$$

The [17] area covered by raindrops or snowflakes in the video image is obviously brighter, and the energy of this pixel will be higher than that of the blocked background pixel. This paper uses local energy to determine the local features of high-frequency images. The [29] local energy is defined as:

$$
E_{A}^{l, k}(p, q)=\Sigma_{m=-1}^{1} \sum_{n=-1}^{1} R(m+2, n+2)\left[I_{A}^{l, k}(p, q)\right]^{2}
$$

The partial area matching degree is similar to the covariance of two partial windows, which represents the similarity. The partial energy matching degree is defined as:

$$
M_{A, B}(p, q)=\frac{2 \sum_{m=-1}^{1} \sum_{n=-1}^{1} R(m+2, n+2) I_{A}^{l, k}(p, q) I_{B}^{l, k}(p, q)}{E_{A}^{l, k}(p, q) \times E_{B}^{l, k}(p, q)}
$$

Given a matching degree threshold $\eta$, its value range is generally $[0.5,1]$. Because the pixel partial energy in the area covered by raindrops and snowflakes is higher than that of the background pixels, when $M_{A, B}(p, q)<\eta$, high-frequency images are used with lower energy, and the fused high-frequency images is defined as:

$$
I_{H F}^{l, k}(p, q)=\left\{\begin{array}{l}
I_{A}^{l, k}(p, q) E_{A}^{l, k} \geq E_{B}^{l, k}(p, q) \\
I_{B}^{l, k}(p, q) \text { others }
\end{array}\right\}
$$

When $M_{A, B}(p, q) \geq \eta$, weights are assigned according to energy. The [26] local weight coefficient is as follows:

$$
\begin{aligned}
& w_{\alpha}(p, q)=\frac{1}{2}+\frac{1}{2}\left(\frac{1-M_{A, B}(p, q)}{1-\eta}\right) \\
& w_{\beta}(p, q)=1-w_{\alpha}(p, q)
\end{aligned}
$$

(3) Reconstructed image

After the fusion is completed, the high-frequency and lowfrequency images are subjected to the inverse transformation of the dual-tree complex wavelet, and the final clear fusion image is obtained by reconstruction. 




(a)

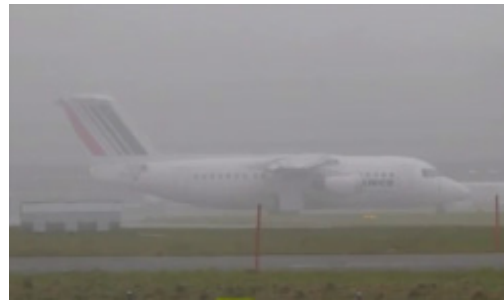

(b)



(c)

Fig. 9. Frames of dynamic video containing large bright regions: (a) the 8th frame (b) the 144th frame (c) the 184th frame.

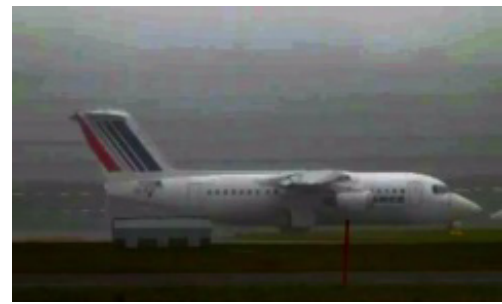

(a)

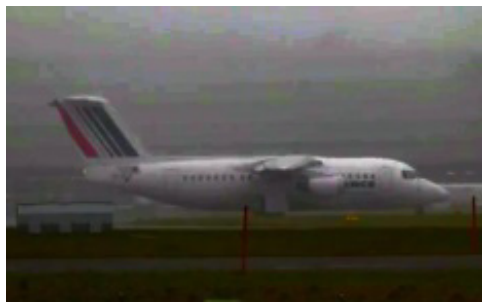

(b)

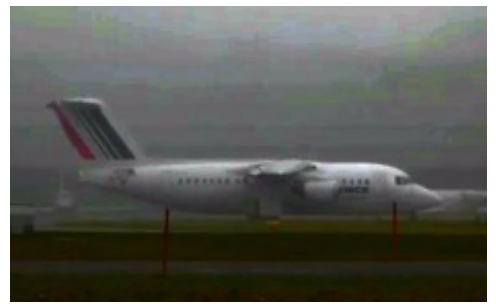

(c)

Fig. 10. Enhanced frames of dynamic video without adaptive tolerance: (a) result of the 8th frame (b) result of the 144th frame (c) result of the 184th frame.

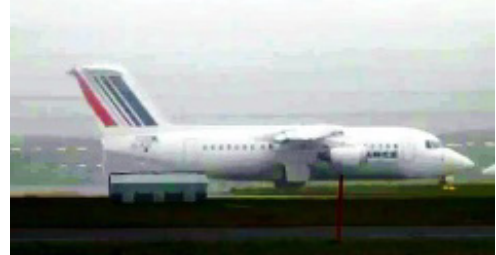

(a)



(b)

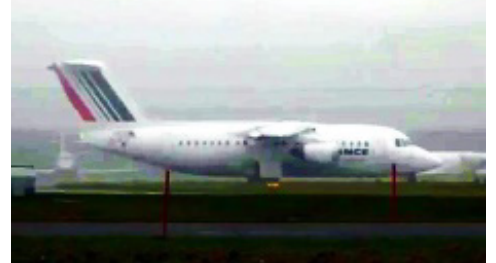

(c)

Fig. 11. Enhanced frames of dynamic video using adaptive tolerance: (a) result of the 8th frame (b) result of the 144th frame (c) result of the 184th frame.

\section{Experimental Results and Discussion}

\section{A. Experimental Results of Image Preprocessing Algorithm Based on Adaptive Tolerance}

The dynamic video is processed in the experiments. These videos contain large bright regions. From the video frame of the dynamic background in Fig. 9, it can be seen that the camera of the video is moving, the aircraft is moving, and the sky and the aircraft belong to a large area of bright areas. In Fig. 10, it can be seen that if these bright areas are not corrected, there will be obvious color distortion in the areas. With the video enhancement based on adaptive tolerance, the corrected results are greatly improved in Fig. 11, and the distortion can be effectively avoided and the image can be clearer. The [12] processing rate of static video is greatly increased and it can reach $25 \mathrm{fps}$.

\section{B. Experimental Effects of Image Matching}

The traditional SIFT feature matching effect is shown in Fig. 12. The improved SIFT feature matching effect proposed in this paper is shown in Fig. 13. The comparison shows that the improved matching method proposed in this paper obtains more correct matching points and higher accuracy.



Fig. 12 The traditional SIFT feature matching effect.

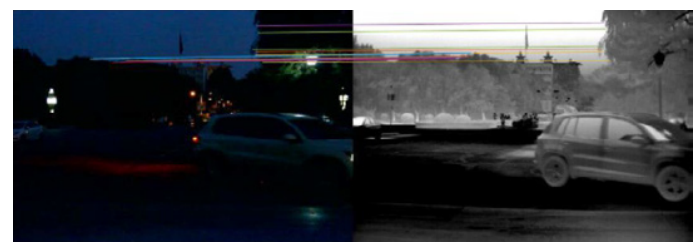

Fig. 13 The improved SIFT feature matching effect.

\section{Experimental Results of Image Fusion}

The [20] first test video frame is an outdoor rainy image. In order to make the result more convincing, the deraining effect of this algorithm is compared and verified with the Dou algorithm. The results are shown in Fig. 14.

The [20] second test video frame is the outdoor rainy image, the desnowing effect of this algorithm is compared and verified with the Dou algorithm. The results are shown in Fig. 15.

It can be seen from the subjective evaluation of Fig. 14(c) and Fig. 15(c) that the effect by the fusion algorithm proposed in this paper is better. It can well remove raindrops or snowflakes from the video image, enhance the image detail information covered by raindrops or snowflakes, and better retain the image edge information.

The experimental results are evaluated from the four objective indicators of the representative evaluation indicators: average gradient, standard deviation, spatial frequency, and entropy, and the fusion results are quantitatively analyzed. The results are shown in Table I and Table II. From the perspective of the objective evaluation indicators, this method is superior to other fusion methods under the traditional fusion rules, and it can effectively retain image details. 


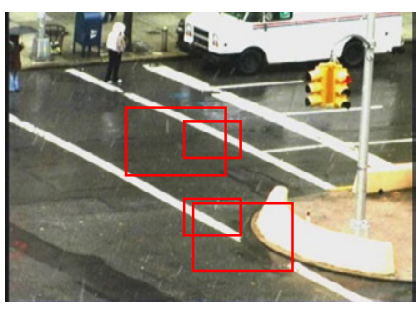

(a)

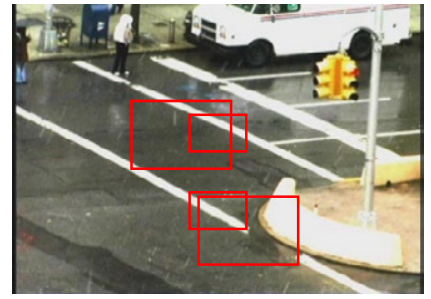

(b)

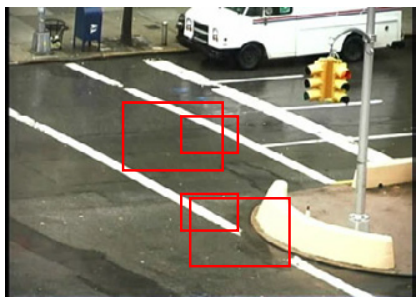

(c)

Fig. 14 The results of the first test video frame. (a)The source image. (b)Dou 's algorithm. (c) Improved algorithm.

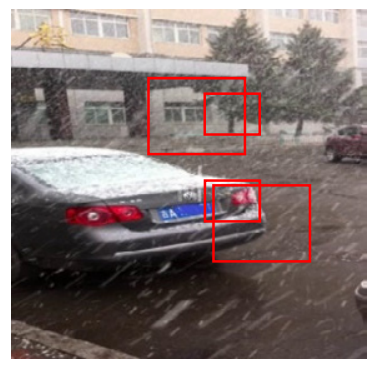

(a)

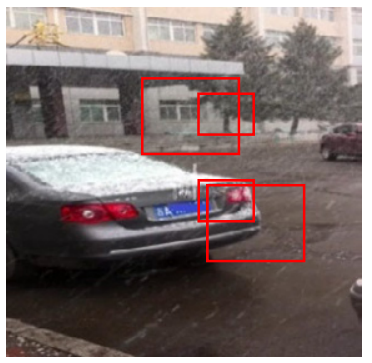

(b)

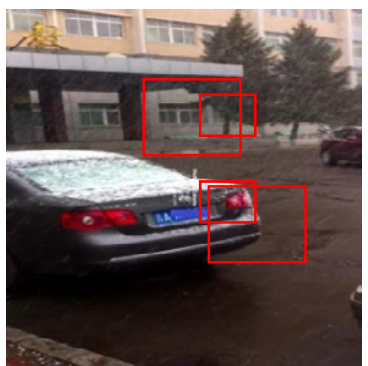

(c)

Fig. 15. The results of the second test video frame: (a) The source image (b) Dou's algorithm (c) Improved algorithm.

TABLE I. The First Test Video Frame

\begin{tabular}{ccccc}
\hline $\begin{array}{c}\text { index } \\
\text { algorithm }\end{array}$ & $\begin{array}{c}\text { Average } \\
\text { Gradient }\end{array}$ & $\begin{array}{c}\text { Standard } \\
\text { Deviation }\end{array}$ & $\begin{array}{c}\text { Spatial } \\
\text { Frequency }\end{array}$ & Entropy \\
\hline Dou's algorithm & 20.7218 & 51.2670 & 40.1258 & 5.1536 \\
\hline $\begin{array}{c}\text { The proposed } \\
\text { algorithm }\end{array}$ & 25.0816 & 60.3450 & 45.8465 & 6.7690 \\
\hline
\end{tabular}

TABLE II. The Second Test Video Frame

\begin{tabular}{ccccc}
\hline $\begin{array}{c}\text { index } \\
\text { algorithm }\end{array}$ & $\begin{array}{c}\text { Average } \\
\text { Gradient }\end{array}$ & $\begin{array}{c}\text { Standard } \\
\text { Deviation }\end{array}$ & $\begin{array}{c}\text { Spatial } \\
\text { Frequency }\end{array}$ & Entropy \\
\hline Dou's algorithm & 21.5478 & 51.3213 & 40.3448 & 5.2014 \\
\hline $\begin{array}{c}\text { The proposed } \\
\text { algorithm }\end{array}$ & 25.3647 & 60.4478 & 45.9657 & 6.9985 \\
\hline
\end{tabular}

\section{Conclusion}

This paper proposes a new algorithm combining dual-tree complex wavelet fusion and adaptive tolerance. The algorithm adopts the fusion rule based on double-tree complex wavelet analysis to improve the quality of the video image under the complex weather background. The adjustment mechanism based on adaptive tolerance can not only solve the problem of distortion in the bright area, but also not make the transmittance be miscompensated, so as to restore a higher quality fused image. The experimental results show that the overall visual effect of the image obtained by the fusion algorithm is better, and it can better recover the details of the image obscured by raindrops or snowflakes.

\section{ACKNOWLEDGMENT}

This work was financially supported by the Scientific and Technological Development Scheme of Jilin Province (No.20170312021ZX).

\section{REFERENCES}

[1] H. Hase, K. Miyake and M. Yoneda, "Real-time snowfall noise elimination," Proceedings 1999 International Conference on Image Processing (Cat. 99CH36348), Kobe, vol. 2, pp. 406-409, 1999, doi:10.1109/ICIP.1999.822927.

[2] K. Garg and S. K. Nayar, "Detection and removal of rain from videos," Proceedings of the 2004 IEEE Computer Society Conference on Computer Vision and Pattern Recognition, 2004. CVPR 2004, Washington, DC, USA, pp. I-I, 2004, doi:10.1109 / CVPR.2004.1315077.

[3] K. Garg and S. K. Nayar, "Vision and Rain," International fournal of Computer Vision 75, pp. 3-27, 2007, doi:10.1007/s11263-006-0028-6.

[4] X. Zhang, H. Li, Y. Qi, W. K. Leow and T. K. Ng, "Rain Removal in Video by Combining Temporal and Chromatic Properties," 2006 IEEE International Conference on Multimedia and Expo, Toronto, pp. 461-464, 2006, doi:10.1109/ICME.2006.262572.

[5] N. Brewer, N. Liu, "Using the shape characteristics of rain to identify and remove rain from video," Lecture Notes in Computer Science, vol. 5342, pp. 451-458, 2008, doi:10.1007/978-3-540-89689-0_49.

[6] X. Zhao, P. Liu, J. Liu, X. Tang, "The application of histogram on rain detection in video," Proceedings of the 11th foint Conference on Information Science, pp. 382-387, 2008, doi:10.2991/jcis.2008.65.

[7] P. Barnum, T. Kanade, \& S. Narasimhan, "Spatio-temporal frequency analysis for removing rain and snow from videos," The First International Workshop on Photometric Analysis for Computer Vision, 1-17 (2008).

[8] P. Barnum, S. Narasimhan, \& T. Kanade, "Analysis of Rain and Snow in Frequency Space," International fournal of Computer Vision 86, pp. 256, 2010, doi:10.1007/s11263-008-0200-2.

[9] J. Bossu, N. Hautière, \& J. Tarel, "Rain or Snow Detection in Image Sequences Through Use of a Histogram of Orientation of Streaks," International Journal of Computer Vision 93, pp. 348-367, 2011, doi: 10.1007/s11263-011-0421-7.

[10] L. Kang, C. Lin and Y. Fu, "Automatic Single-Image-Based Rain Streaks Removal via Image Decomposition," in IEEE Transactions on Image Processing, vol. 21, no. 4, pp. 1742-1755, 2012, doi:10.1109/ TIP.2011.2179057.

[11] L. Kang, C. Lin and Y. Lin, "Self-learning-based rain streak removal for image/video," 2012 IEEE International Symposium on Circuits and Systems (ISCAS), Seoul, pp. 1871-1874, 2012, doi:10.1109/ISCAS.2012.6271635.

[12] L. Yu, Y. Piao, X. Yan, "Video Defogging Based on Adaptive Tolerance," TELKOMNIKA: Indonesian Journal of Electrical Engineering, vol. 10, pp. 1644-1654, 2012, doi:10.11591/telkomnika.v10i7.1556.

[13] A. E. Abdel-Hakim, "A Novel Approach for Rain Removal from Videos Using Low-Rank Recovery," 2014 5th International Conference on 
Intelligent Systems, Modelling and Simulation, Langkawi, pp. 351-356, 2014, doi:10.1109 / ISMS.2014.161.

[14] J. Kim, J. Sim and C. Kim, "Video Deraining and Desnowing Using Temporal Correlation and Low-Rank Matrix Completion," in IEEE Transactions on Image Processing, vol. 24, no. 9, pp. 2658-2670, 2015, doi:10.1109/TIP.2015.2428933.

[15] W. Ren, J. Tian, Z. Han, A. Chan and Y. Tang, "Video Desnowing and Deraining Based on Matrix Decomposition," 2017 IEEE Conference on Computer Vision and Pattern Recognition (CVPR), Honolulu, HI, pp. 28382847, 2017, doi:10.1109/CVPR.2017.303.

[16] X. Zheng, Y. Liao, W. Guo, X. Fu, X. Ding, "Single-Image-Based rain and snow removal using multi-guided filter," 20th International Conference on Neural Information Processing (ICONIP). Springer Berlin Heidelberg, vol. 8228, pp. 258-265, 2013, doi:10.1007/978-3-642-42051-1_33.

[17] Z. Wang, "Method of Removing Rain (Snow) from Video Images Based on Wavelet Fusion," fournal of BeiHua University (Natural Science), vol. 19, no. 1, pp. 136-140, 2018.

[18] S. G. Narasimhan and S. K. Nayar, "Contrast restoration of weather degraded images," in IEEE Transactions on Pattern Analysis and Machine Intelligence, vol. 25, no. 6, pp. 713-724, June 2003 doi:10.1109/ TPAMI.2003.1201821.

[19] K. He, J. Sun, X. Tang, "Guided image filtering," European Conference on Computer Vision 2010, Part I, Lecture Notes in Computer Science, vol. 6311, pp. 1-14, doi:10.1007/978-3-642-15549-9_1.

[20] L. Dou, D. Xu, J. Li, "Image Inpainting Based on Dual-tree Complex Wavelet Transform," Computer Science, vol. 44, no. 6, pp. 180-191, 2017.

[21] J. Du, S. Chen, "Adaptive PCNN Image Fusion Algorithm Based on Double Tree Complex Wavelet Transform," Infrared Technology, vol. 40, no. 10, pp. 1002-1007, 2018.

[22] W. Yang, Q. Chai, L. Wang, "Multi-focus image fusion method based on double-tree complex wavelet transform," Electro-Optic Technology Application, vol. 24, no. 3, pp. 59-62, 2009.

[23] F. Yang, L. Zheng, "New feature extraction algorithm based on SIFT, Applied Science and Technology, vol. 1, no. 1, pp. 1-5, 2018.

[24] Y. Xiang, H. Qin, J. Li et al., "Multi-focus image fusion using a guidedfilter-based difference image," Applied Optics, vol. 55, no. 9, pp. 2230-2239, 2016, doi:10.1364/AO.55.002230.

[25] J. Li, Y. Yang, "An Improved MLESAC Algorithm for Estimating Fundamental Matrix," Computer Engineering, vol. 38, no. 19, pp. 215-217, 2012.

[26] M. R. Metwalli, A. H. Nasr, O. S. Farag Allah and S. El-Rabaie, "Image fusion based on principal component analysis and high-pass filter," 2009 International Conference on Computer Engineering \& Systems, Cairo, pp. 63-70, 2009, doi:10.1109/ICCES.2009.5383308.

[27] S. Lu, L. Zou, X. Shen, W. Wu, W. Zhang, "Multi-spectral remote sensing image enhancement method based on PCA and IHS transformations," fournal of Zhejiang University-SCIENCE A, vol. 12, pp. 453-460, 2011, doi:10.1631/jzus.A1000282.

[28] X. Wang, Y. Zhou, "Infrared and visible fusion in undecimated dual-tree complex wavelet domain," Computer Engineering and Design, vol. 38, no.3, pp. 730-734, 2017.

[29] F. Wu, H. Li, "Image Fusion Algorithm Based on NSCT and PCA," Aeronautical computing technique, vol. 45, no. 3, pp. 48-50, 2015, doi:10.12968/prtu.2015.45.48.

[30] J. Zang, G. Ren, J. Dong, Y. Piao, S. Jim, "Removal of rain video based on temporal intensity and chromatic constraint of raindrops," Evolutionary Intelligence, vol. 12, pp. 349-355, 2019, doi:10.1007/s12065-018-0185-x.

[31] J. Zang, G. Ren, Y. An, Y. Piao, "Removal of rain from video based on dualtree complex wavelet fusion," fournal of Intelligent \& Fuzzy Systems, vol. 38, no. 1, pp. 105-113, 2020, doi:10.3233/JIFS-179385.

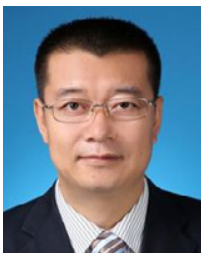

\section{Jingfeng Zang}

Jingfeng Zang, associate professor of Changchun University of Science and Technology, received a bachelor's degree from Changchun University of Science and Technology in 2000, a master's degree from Changchun University of Science and Technology in 2004, and a doctorate from Changchun University of Science and Technology in 2010. From November 2013 to September 2014, he was a visiting scholar at the Norwegian University of Science and Technology, mainly engaged in the development and application of heterogeneous multicore embedded systems. In recent years, he has published more than 10 academic papers on video and image processing, including 6 papers in EI, 3 core papers, 1 utility model patent, and won 1 third prize for scientific and technological progress in Jilin Province. As a backbone, he participated in 1 National Natural Science Foundation project, 1 Ministry of Science and Technology project, 4 Jilin Science and Technology Department project, Changchun City Science and Technology Department project, Jilin Province Education Department project, etc., mainly responsible for hardware system design and The hardware transplantation of software algorithms has accumulated rich scientific research experience, and made great breakthroughs in video image processing related theories and key technologies. At present, he is mainly engaged in the research of video image processing technology, video surveillance, intelligent information processing and the transformation of technological achievements of joint enterprises.

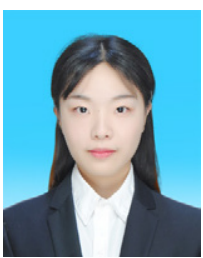

Ningxue Xu

Ningxue Xu, graduated from China in 2019 with a bachelor's degree in electronic information engineering from Anyang Institute of Technology. Currently studying for a master's degree in electronic science and technology in the School of Electronic Information Engineering of Changchun University of Science and Technology. Mainly research sensing and signal processing technology, convolutional neural network technology, video security monitoring technology, image registration, fusion, matching technology, etc. Good at researching multifocus image fusion technology based on deep learning.



Rui Liu

Rui Liu, graduated from the School of Electronic Information Engineering, Changchun University of Science and Technology in 2019 with a bachelor's degree in engineering, Jilin, China. Currently studying for a master's degree in the School of Electronic Information Engineering, Changchun University of Science and Technology, majoring in electronic science and technology. The main research directions are sensing and signal processing technology, video image processing technology, video security monitoring technology, and deep learning. Good at researching face state detection and evaluation based on deep learning.

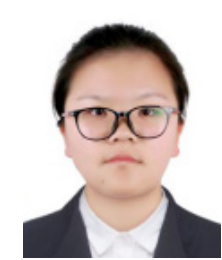

Yuhuan Shi

Yuhuan Shi, graduated from Jilin, China in 2019 with a bachelor's degree in electronic information engineering from Beihua University. He is currently a master's student at Changchun University of Science and Technology. The major is electronic science and technology. Current research interests include image processing or video processing based on deep learning, surveillance video analysis, and video content understanding. The specific research direction is pedestrian detection and pedestrian re-identification based on deep learning. Good at researching pedestrian re-identification technology and pedestrian detection technology based on deep learning. 